\title{
Case Report \\ A Case of Transverse-sigmoid Sinus Dural Arteriovenous Fistula Treated by Transarterial and Transvenous Embolization via the Balloon Microcatheter with Overinflated Balloon Protection of the Torcula
}

Hajime Yabuzaki, ${ }^{1}$ Tomoaki Terada, ${ }^{2}$ Hisato Ikeda, ${ }^{3}$ Michiari Kawamo, ${ }^{3}$ Akira Wada, ${ }^{1}$ Sadayoshi Nakayama, ${ }^{1}$ Tomomi Yoshiyama, ${ }^{1}$ Eisuke Hirose, ${ }^{4}$ Yuuta Kawauchi, ${ }^{5}$ and Yoshifumi Yamaguchi ${ }^{5}$

Objective: The objectives of treating dural arteriovenous fistula (dAVF) are to achieve complete occlusion of the arteriovenous (AV) shunt and restoration of anterograde venous flow. We report a patient with complex dAVF who was managed by double overinflated balloon protection of the torcula and percutaneous transluminal angioplasty (PTA) for the occluded venous sinus.

Case Presentation: A 62-year-old woman was admitted with gradually worsening nausea and vomiting, as well as progressive memory disturbance and left hemiparesis. Head MRI/MRA demonstrated a dAVF involving the region from the right sigmoid sinus (SS) to transverse sinus (TS) along with occlusion of the left TS and right proximal SS. There was associated dilatation of the right TS, torcula, and superior sagittal sinus (SSS). The intracranial venous circulation was impaired, with venous reflux draining into the deep cerebral veins as well as the cortical veins. Transarterial embolization (TAE) and transvenous embolization (TVE) were performed with double overinflated balloon protection of the torcula, and shunt flow was completely abolished. The occluded sinus was recanalized by PTA. Her symptoms gradually improved after treatment.

Conclusion: In this patient, double overinflated balloon protection was effective for maintaining the torcula.

Keywords > dural arteriovenous fistula, balloon protection, venous sinus, transarterial embolization

${ }^{1}$ Department of Neurosurgical, Ebara Hospital, Tokyo Metropolitan Health and Hospitals Corporation, Tokyo, Japan

${ }^{2}$ Department of Neurosurgical, Showa University Fujigaoka Hospital, Yokohama, Kanagawa, Japan

${ }^{3}$ Department of Neurosurgical, Showa University Toyosu Hospital, Tokyo, Japan

${ }^{4}$ Department of Neurosurgical, Showa University Hospital, Tokyo, Japan

${ }^{5}$ Department of Neurosurgical, Showa University Northern Yokohama Hospital, Yokohama, Kanagawa, Japan

Received: August 2, 2018; Accepted: July 2, 2019 Corresponding author: Hajime Yabuzaki. Department of Neurosurgical, Ebara Hospital, Tokyo Metropolitan Health and Hospitals Corporation, 4-5-10 Higashiyukigaya, Ota-ku, Tokyo 145-0065, Japan Email: yabuzaki-kyt@umin.ac.jp

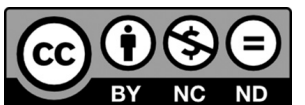

This work is licensed under a Creative Commons Attribution-NonCommercialNoDerivatives International License.

(C)2019 The Japanese Society for Neuroendovascular Therapy

\section{Introduction}

In patients with a dural arteriovenous fistula (dAVF) involving the venous sinuses accompanied by cortical venous reflux, treatment aims to achieve complete occlusion of the arteriovenous (AV) shunt, and sinus packing via the transvenous approach is generally performed unless embolization $^{1)}$ is applicable. In Japan, Onyx became available during this fiscal year, increasing the possibility of treating dAVF by transarterial embolization (TAE) in addition to transvenous embolization (TVE). ${ }^{2-4)}$ Due to the availability of this new method, the objective of treating dAVF may be revised to achieving complete occlusion of the shunt along with preservation or restoration of normal venous return. We report a patient with dAVF in whom the AV shunt was occluded while inflating two balloons to prevent migration of embolic material into the venous sinuses, 

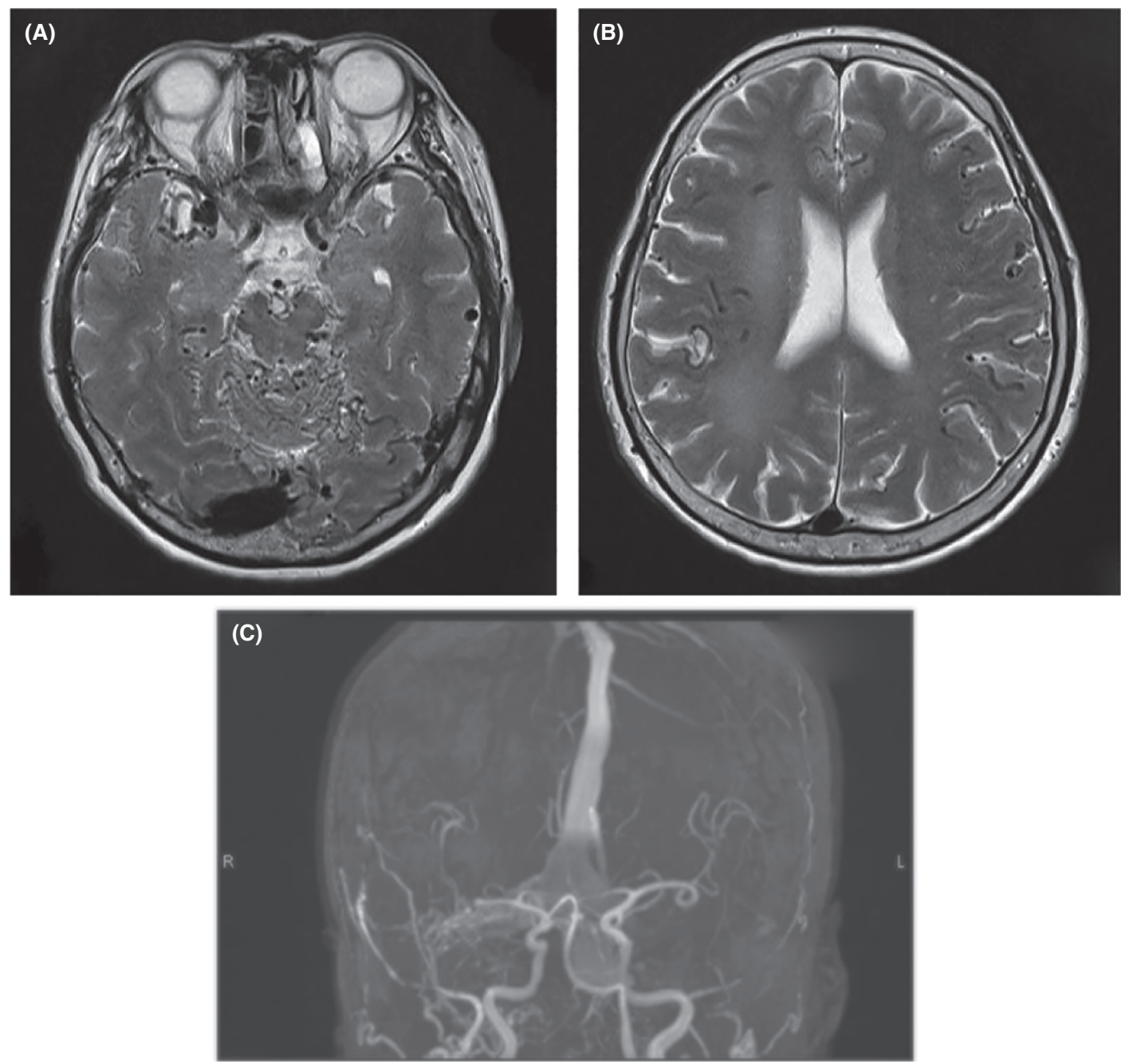

Fig. 1 (A and B) Axial T2 images show hyperintensity in the white matter of the right hemisphere, as well as dilation of the right TS and the cortical veins. (C) MRA reveals dilation of the torcula and the SSS, along with the presence of arteriovenous shunts and cortical venous reflux. TS: transverse sinus; SSS: superior sagittal sinus

followed by percutaneous transluminal angioplasty (PTA) of the occluded sinus to restore normal venous return, resulting in curative treatment of the dAVF.

\section{Case Presentation}

A 62-year-old woman complained of nausea and vomiting for 3 months, as well as bradykinesia and memory disturbance for 1 month. Her symptoms progressed to left hemiparesis and dressing apraxia. Head MRI was performed and a dAVF was suspected. Therefore, the patient was referred to our hospital for further management and was urgently admitted. On admission, her consciousness was clear and she was oriented. She complained of persistent nausea, and paresis (MMT4/5) of the left upper and lower limbs was observed. After admission, her nausea gradually became worse and her paralysis progressed (MMT3/5), along with difficulty eating and disturbance of consciousness (E3V5M6/GCS).

On head MRI/MRA, cerebral surface veins were markedly dilated throughout the supratentorial and infratentorial regions, as was the superior sagittal sinus (SSS) and the right transverse sinus (TS). There was marked edema of the white matter in the entire right cerebral hemisphere. No dilatation of the deep venous system was noted. MRA visualized dilation of the SSS and right TS (Fig. 1).

Based on these findings, dAVF was suspected and digital subtraction angiography (DSA) was performed.

\section{Digital subtraction angiography}

A dAVF was detected with multiple feeding vessels, including the right tentorial artery (on right internal carotid artery angiography), the bilateral occipital arteries, bilateral 

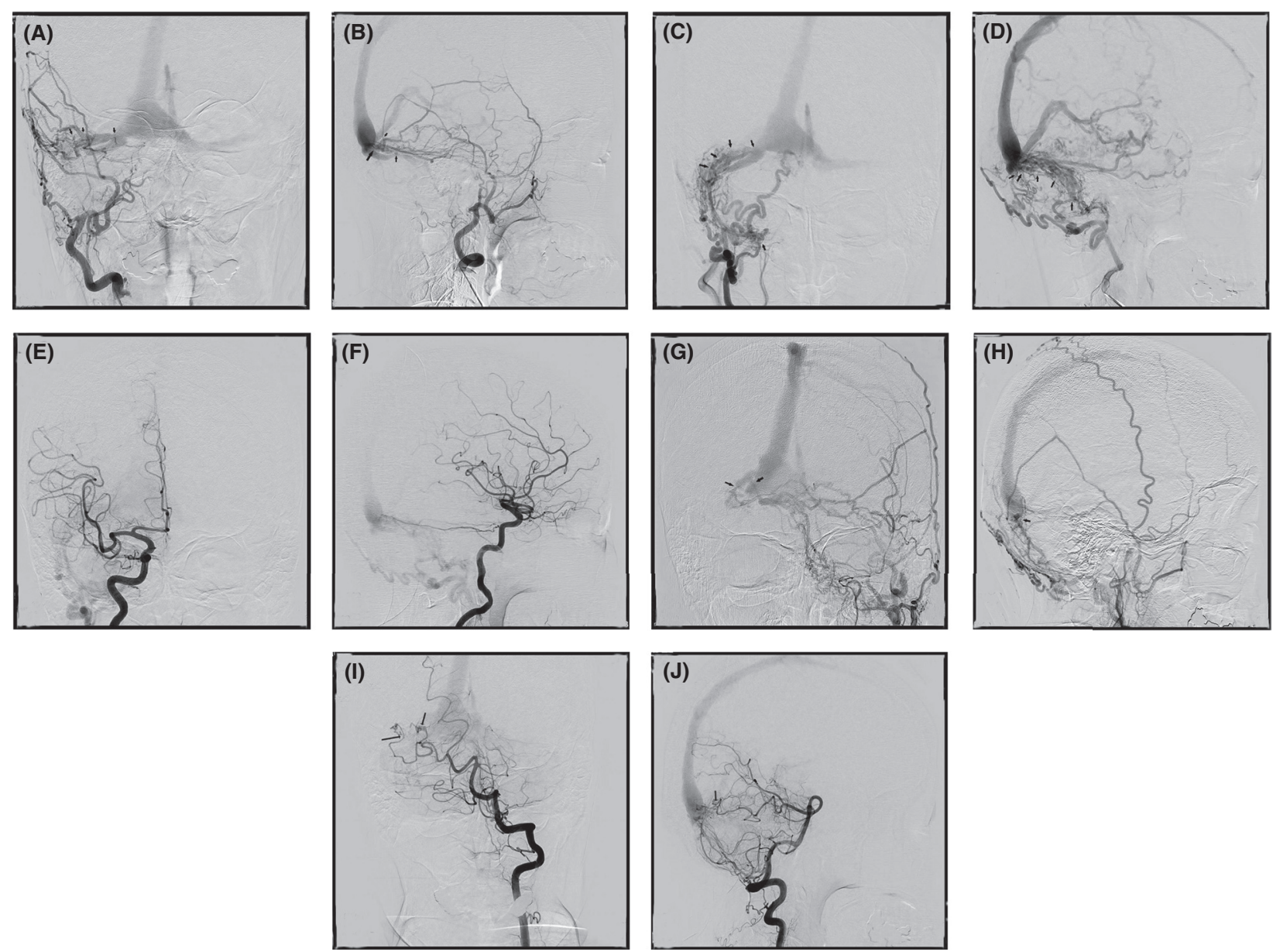

Fig. 2 (A and B) Right distal ECAG (A, frontal view; B, lateral view) shows the dAVF involving the right TS and right SS fed by the right middle meningeal artery. (C and D) Right occipital angiogram (C, frontal view; D, lateral view) displays AV shunts (arrows) to the right SS and right TS. Occlusion of the distal end of the left TS can also be seen. ( $E$ and $\mathbf{F}$ ) Right ICAG reveals a feeder from the right tentorial artery. ( $\mathbf{G}$ and $\mathbf{H}$ ) Left ECAG shows the dAVF fed by the left middle meningeal artery and left occipital artery. There are AV shunts close to the torcula and another AV shunt on the proximal side of the right TS. (I and J) Left VAG displays the dAVF supplied by the left posterior meningeal artery and by pial feeders from the left posterior cerebral artery (arrows). AV: arteriovenous; dAVF: dural arteriovenous fistula; ECAG: external carotid artery angiography; ICAG: internal carotid artery angiography; SS: sigmoid sinus; TS: transverse sinus; VAG: vertebral artery angiography

middle meningeal arteries, and left posterior meningeal artery (on bilateral external carotid artery angiography), and the left posterior cerebral artery (on left vertebral artery angiography) (Fig. 2). Several shunts were present near the confluence of the right TS with the torcula, including shunts to the right transverse-sigmoid sinus (SS) junction (TS junction) and the right SS. The shunt from the medial side of the right TS to the right side of the torcula also received blood from the posterior convexity branch of the left middle meningeal artery, left occipital artery, and posterior meningeal artery. Both cortical venous reflux and reflux from the torcula into the deep venous system, including the sinus rectus and vein of Galen, were observed. Since the central part of the right SS was occluded and the left TS was occluded in the region near the SS, venous return flowed backward in the SSS and then retrogradely through the cerebral surface veins to eventually reach the left SS via the left vein of Labbe.

Based on these DSA findings, a symptomatic dAVF (right transverse-SS, Borden type II, Cognard II a $+\mathrm{b}$ ) was diagnosed and endovascular treatment was performed.

\section{Endovascular treatment (TVE and TAE)}

Under general anesthesia, a $7 \mathrm{Fr}$ sheath $(25 \mathrm{~cm})$ was inserted into the right femoral artery, after which a 7 Fr guiding catheter (ENVOY, Johnson \& Johnson, Miami, FL, USA) and 4.2 Fr intermediate catheter (FUBUKI, Asahi Intecc Co., Ltd., Aichi, Japan) were advanced coaxially to the right external carotid artery. The left internal jugular vein was punctured directly and a $7 \mathrm{Fr}$ sheath $(25 \mathrm{~cm})$ was placed to allow insertion of a guide wire (Radifocus; Terumo, Tokyo, Japan), along with coaxial advancement of a $7 \mathrm{Fr}$ guiding catheter 

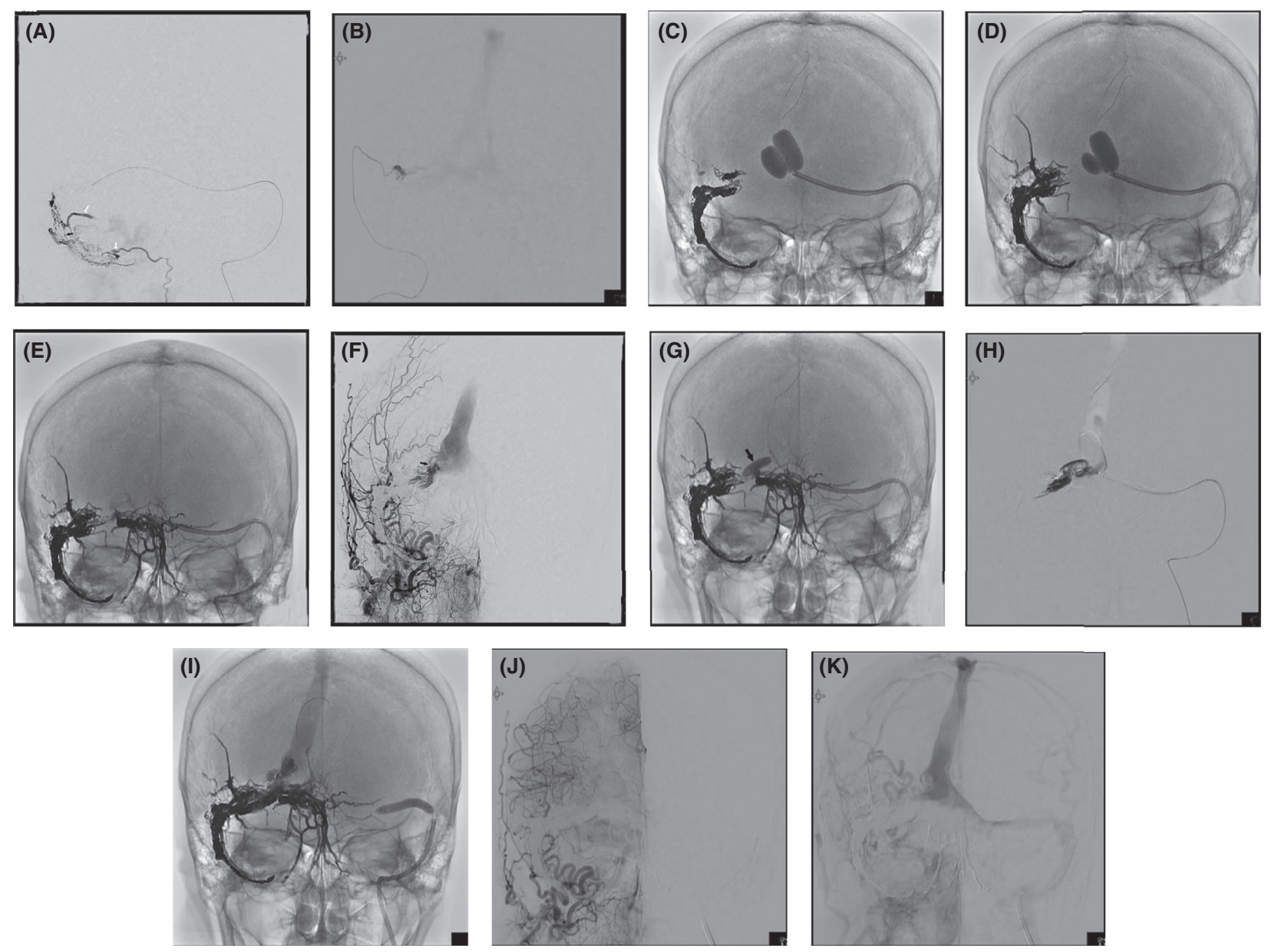

Fig. 3 (A) (Frontal view) During TVE (coil embolization) of the right transverse-SS, tip injection from the microcatheter (in the right SS) shows residual venous flow (white arrows). Arrowhead indicates the tip of the microcatheter. (B) (Frontal view) Right MMAG from the microcatheter without balloon protection. (C) Fluoroscopy showing double overinflated balloon catheters placed at the torcula. (D) (Frontal view) TAE with ONYX from the right middle meningeal artery under double balloon protection to prevent migration into the torcula. (E) (Frontal view) TAE with ONYX from the left middle meningeal artery under double balloon protection to prevent migration into the torcula. (F) (frontal view) Right ECAG after TAE via the right and left middle meningeal arteries. Residual shunt is observed (arrow). (G) (Frontal view) Fluoroscopy during inflation of the double-lumen balloon microcatheter (arrow) inserted into the residual AV shunt from the left TS. (H) (Frontal view) ONYX cast injected from a Scepter C balloon catheter placed to the AV shunt via the right TS. (I) (Frontal view) Fluoroscopy during balloon angioplasty of the left TS. ( $\mathbf{J}$ and $\mathbf{K}$ ) (Frontal view) Right CCAG shows complete occlusion of the dAVF and restoration of antegrade venous flow from the SSS to the left TS. AV: arteriovenous; CCAG: common carotid artery angiography; dAVF: dural arteriovenous fistula; ECAG: external carotid artery angiography; MMAG: middle meningeal artery angiography; SS: sigmoid sinus; SSS: superior sagittal sinus; TAE: transarterial embolization; TVE: transvenous embolization; TS: transverse sinus

(FUBUKI) and 4 F catheter (Tempo; Cordis Medical, Bridgewater, NJ, USA). The guiding catheter was retrogradely advanced through the occlusion in the left TS to a site near the torcula. Using a microcatheter (Headway 17, Microvention, Inc., Tustin, CA, USA) and guide wire (CHIKAI-14, Asahi Intecc Co., Ltd), the catheter was advanced to the central part of the SS through the venous sinus compartment from the end of the right TS to the right SS, after which TVE was done by retrieving the catheter while placing coils from the central part of the SS to near the TS-SS junction. When contrast medium was injected from the tip of the microcatheter in the SS, residual reflux into the cortical veins was observed (Fig. 3A). Therefore, the right SS was completely occluded by injection of Onyx 18 liquid embolic agent (ethylene vinyl alcohol copolymer; Covidien/ ev3, Irvine, CA, USA) via the same microcatheter. Then, the microcatheter (Headway 17) was inserted into the shunt near the TS junction from the venous side, and the sinus was occluded with coils. However, the shunt fed by the bilateral middle meningeal arteries and left occipital artery still remained. It was considered that complete occlusion of this shunt by TVE with coils alone would be difficult because of requiring many coils and taking considerable time, so it was decided to perform TAE. 
A Scepter C $4.0 \mathrm{~mm} \times 10 \mathrm{~mm}$ (Microvention, Inc.) was guided to the periphery (very close to the shunt) of the posterior convexity branch of the right middle meningeal artery, and angiography was performed. The right TS was immediately visualized through the shunt, raising concern about early outflow of injected Onyx into the sinus (Fig. 3B). Therefore, 2 SHOURYU $7.0 \mathrm{~mm} \times 7 \mathrm{~mm}$ balloons were inflated near the torcula (Fig. 3C) before Onyx18 was injected for TAE. By performing TAE with balloon protection, reflux of Onyx into the other feeders and inflow into the sinus could be prevented (Fig. 3D). After confirming the disappearance of shunt blood flow from the right middle meningeal artery, TAE was done via the left middle meningeal artery for the residual shunt on the right side of the torcula. A Headway 17 (Microvention, Inc.) was guided to the periphery (very close to the shunt) of the posterior convexity branch of the left middle meningeal artery, after which two SHOURYU $7.0 \mathrm{~mm} \times 7 \mathrm{~mm}$ balloons were inflated near the torcula and Onyx 18 was injected using the plug and push method (Fig. 3E). When angiography was performed at this stage, a residual shunt was detected near the medial part of the right TS on the right lateral side of the torcula (Fig. 3F). A Scepter C $4.0 \mathrm{~mm} \times 10 \mathrm{~mm}$ was inserted into the shunt from the torcular side, and the balloons were inflated to block blood flow to the torcula (Fig. 3G). Then retrograde injection of Onyx was done from the venous side to form an Onyx cast that occluded the shunt at the site of catheter insertion, and injection was stopped when slight reflux of Onyx into the sinus side was noted (Fig. 3H). Angiography showed that shunt blood flow was completely absent. Finally, PTA was done for the occluded region of the left TS using a $5.0 \mathrm{~mm} \times 40 \mathrm{~mm}$ Sterling balloon (Boston Scientific, Marlborough, MA, USA) (Fig. 3I). The left TS was sufficiently dilated, restoring a normal venous return route. Although dilatation of cortical veins and venous congestion remained, restoration of normal venous flow from the cortical veins to the SSS and TS was confirmed, and treatment was completed (Fig. $\mathbf{3} \mathbf{J}$ and $\mathbf{K}$ ).

\section{Postoperative course}

No cerebral infarction was detected on postoperative MRI. Left conjugate deviation was noted after the patient woke from anesthesia and medication was administered because of concern about seizures. However, conjugate deviation resolved on the following day and did not recur. An antiplatelet drug (clopidogrel $75 \mathrm{mg} / \mathrm{d}$ ) was administered to prevent re-occlusion of the left TS. Immediately after treatment, MRI showed improvement of dilatation of the cortical veins and edema of the white matter, and both the level of consciousness and left hemiplegia also gradually improved. Her consciousness became clear and independent walking became possible, although there was residual mild left hemiplegia. The patient was transferred to a rehabilitation hospital (modified Rankin Scale [mRS]2) at 20 days after the procedure.

\section{Discussion}

In the present patient, we performed Onyx injection combined with balloon occlusion on the arterial side, TAE by the plug and push method, and embolization by injection of Onyx into the mass of coils. In addition, the following new methods were employed: (1) preservation of the torcula by in-line inflation of 2 SHOURYU $7.0 \mathrm{~mm} \times 7 \mathrm{~mm}$ balloons and (2) insertion of a double-lumen balloon through the torcula to occlude a small residual shunt opening in the right TS, after which Onyx was injected retrogradely into the shunt from the venous side to achieve complete occlusion.

In the past, dAVF accompanied by cortical venous reflux was mainly treated by sinus packing. However, new treatment methods have been reported after Onyx was developed, such as TAE with injection of Onyx while occluding the feeder artery with a balloon catheter, ${ }^{4-10)}$ TAE with control of blood flow by inflating a balloon in the sinus, ${ }^{7,11)}$ and various methods for injection of Onyx, including the pressure cooker technique using a double-lumen balloon catheter. ${ }^{12,13)}$ It is now possible to select treatment from combinations of various devices and liquid embolic materials, leading to attempts to achieve occlusion of the dAVF while conserving the sinus.

Many patients are treated by sinus packing alone, ${ }^{14)}$ since it is an effective curative option for dAVF. However, recurrence at another site several months after sinus packing has been reported. ${ }^{15,16)}$ Regarding such recurrence, it has been reported that dAVF is caused by occlusion of a venous sinus accompanied by elevation of venous pressure and neovascularization. ${ }^{17)}$ Ectopic recurrence of dAVF is considered to be due to elevation of venous pressure induced by sinus stenosis after treatment and may be prevented by recanalization of the sinus, ${ }^{18)}$ suggesting that elevation of venous pressure is involved in the development and progression of dAVF. Based on these reports, we considered that treatment of dAVF and prevention of recurrence could be more reliably achieved by recanalization the occluded sinus and planned to achieve this. Although recanalization of the right 
TS-SS was difficult in the present case, reconstruction of the left sinus restored antegrade venous return and sinus plasty was also performed to improve venous return. ${ }^{19)}$ In the present patient, a SHOURYU $7.0 \mathrm{~mm} \times 7 \mathrm{~mm}$ balloon was used to prevent migration of Onyx into the sinus during TAE because no balloon catheter specifically for sinus occlusion is currently available in Japan. When a SHOURYU $7.0 \mathrm{~mm} \times 7 \mathrm{~mm}$ balloon is overinflated, it expands to about $12 \times 30 \mathrm{~mm}$ and is large enough to occlude the TS-SS. ${ }^{10}$ However, this was not sufficient to occlude the torcula and overinflation of two balloons was required. Inflation on the venous side was adjusted while performing angiography before Onyx injection and occlusion was maintained for as short a time as possible.

According to a previous report on treatment while inflating a balloon in the sinus, the sinus affected by a dAVF was occluded with a balloon and the feeder was treated by TAE using Onyx and a double-lumen balloon catheter, achieving complete occlusion of the shunt. In our patient, the affected sinus was not occluded, but the outflow path was temporarily blocked and penetration of the other feeders was achieved, as previously reported, suggesting that this was useful to increase the effectiveness of TAE. The multiple balloon method employed in this patient may be useful when the dAVF drains to a site near the torcula and preservation of the sinus and torcula using a normal-size balloon during TAE is considered to be difficult due to likely migration of the embolic material.

\section{Conclusion}

We reported a patient in whom a dAVF was occluded by performing TAE and TVE with coils and Onyx while protecting the torcula with two balloons, followed by PTA of the occluded sinus to re-establish normal venous return. Devising various balloon techniques may help to develop a procedure for dAVF that allows preservation and reconstruction of the sinus.

\section{Disclosure Statement}

There is no conflict of interest to be disclosed in this report.

\section{References}

1) Mironov A: Selective transvenous embolization of dural fistulas without occlusion of the dural sinus. AJNR Am J Neuroradiol 1998; 19: 389-391.
2) van Rooij WJ, Sluzewski M: Curative embolization with Onyx of dural arteriovenous fistulas with cortical venous drainage. AJNR Am J Neuroradiol 2010; 31: 1516-1520.

3) Guedin P, Gaillard S, Boulin A, et al: Therapeutic management of intracranial dural arteriovenous shunts with leptomeningeal venous drainage: report of 53 consecutive patients with emphasis on transarterial embolization with acrylic glue. J Neurosurg 2010; 112: 603-610.

4) Kim JW, Kim BM, Park KY, et al: Onyx embolization for isolated type dural arteriovenous fistula using a dual-lumen balloon catheter. Neurosurgery 2016; 78: 627-636.

5) Kerolus MG, Chung J, Munich SA, et al: An Onyx tunnel: reconstructive transvenous balloon-assisted Onyx embolization for dural arteriovenous fistula of the transverse-sigmoid sinus. J Neurosurg 2018; 129: 922-927.

6) Kim SH, Kim TG, Kong MH: Transarterial balloonassisted Onyx embolization of intracranial arteriovenous malformations using a dual-lumen balloon microcatheter: two case reports. J Cerebrovasc Endovasc Neurosurg 2017; 1919: 223-230.

7) Shi ZS, Loh Y, Duckwiler GR, et al: Balloon-assisted transarterial embolization of intracranial dural arteriovenous fistulas. J Neurosurg 2009; 110: 921-928.

8) Kim ST, Jeong HW, Seo J: Onyx embolization of dural arteriovenous fistula, using scepter $\mathrm{C}$ balloon catheter: a case report. Neurointervention 2013; 8: 110.

9) Spiotta AM, Miranpuri AS, Vargas J, et al: Balloon augmented Onyx embolization utilizing a dual lumen balloon catheter: utility in the treatment of a variety of head and neck lesions. J Neurointerv Surg 2014; 6: $547-555$

10) Piechowiak E, Zibold F, Dobrocky T, et al: Endovascular treatment of dural arteriovenous fistulas of the transverse and sigmoid sinuses using transarterial balloon-assisted embolization combined with transvenous balloon protection of the venous sinus. AJNR Am J Neuroradiol 2017; 38: 1984-1989.

11) Zhang Y, Li Q, Huang QH: Embolization of a superior sagittal sinus dural arteriovenous fistula under intrasinus balloon protection: a case report. Interv Neuroradiol 2015; 21 : 94-100.

12) Chapot R, Stracke P, Velasco A, et al: The pressure cooker technique for the treatment of brain AVMs. J Neuroradiol 2014; 41: 87-91.

13) Dabus G, Linfante I, Martínez-Galdámez M: Endovascular treatment of dural arteriovenous fistulas using dual lumen balloon microcatheter: technical aspects and results. Clin Neurol Neurosurg 2014; 117: 22-27.

14) Carlson AP, Alaraj A, Amin-Hanjani S, et al: Endovascular approach and technique for treatment of transversesigmoid dural arteriovenous fistula with cortical reflux: the 
importance of venous sinus sacrifice. $J$ Neurointerv Surg 2013; 5: 566-572.

15) Kubo M, Kuwayama N, Hirashima Y, et al: Dural arteriovenous fistulae developing at different locations after resolution of previous fistulae: Report of three cases and review of the literature. AJNR Am J Neuroradiol 2002; 23:787-789.

16) Kurata A, Suzuki S, Iwamoto K, et al: New development of a dural arteriovenous fistula (AVF) of the superior sagittal sinus after transvenous embolization of a left sigmoid sinus dural AVF. Case report and review of the literature. Interv Neuroradiol 2006; 12: 363-368.
17) Terada T, Higashida RT, Halbach VV, et al: Development of acquired arteriovenous fistulas in rats due to venous hypertension. J Neurosurg 1994; 80: 884-889.

18) Haraguchi K, Matsumoto $Y$, Kondo R, et al: Percutaneous transluminal sinus angioplasty for transverse sinus-sigmoid sinus stenosis associated with recurrent dural arteriovenous fistula: case report. JNET 2010; 4: 33-39.

19) Strauss I, Frolov V, Buchbut D, et al: Critical appraisal of endovascular treatment of brain arteriovenous malformation using Onyx in a series of 92 consecutive patients. Acta Neurochir (Wien) 2013; 155: 611-617. 